\title{
Pharmacokinetics and safety of oral glyburide in dogs with acute spinal cord injury
}

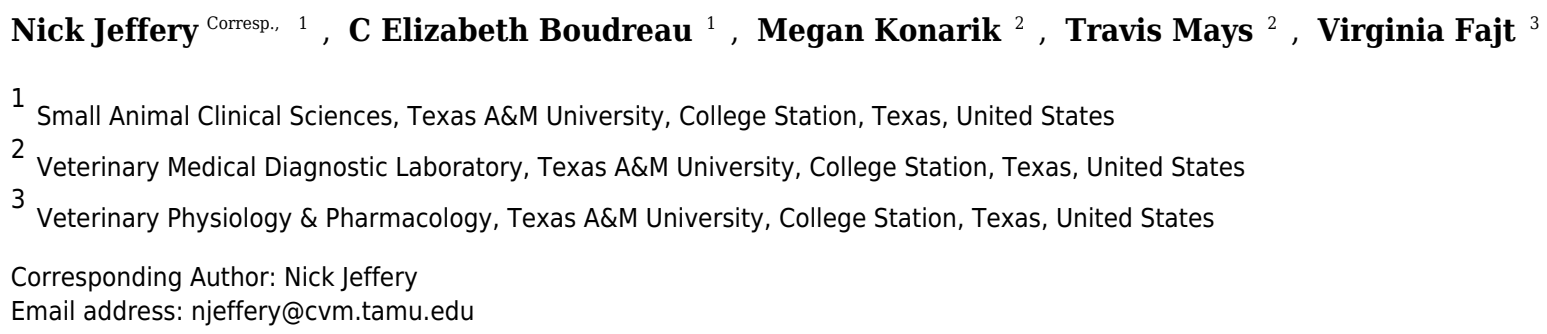

Background. Glyburide (also known as glibenclamide) is effective in reducing the severity of tissue destruction and improving functional outcome after experimental spinal cord injury in rodents and so has promise as a therapy in humans. There are many important differences between spinal cord injury in experimental animals and in human clinical cases, making it difficult to introduce new therapies into clinical practice. Spinal cord injury is also common in pet dogs and requires new effective therapies, meaning that they can act as a translational model for the human condition while also deriving direct benefits from such research. In this study we investigated the pharmacokinetics and safety of glyburide in dogs with clinical spinal cord injury. Methods. We recruited dogs that had incurred an acute thoracolumbar spinal cord injury within the previous 72 hours. These had become acutely non-ambulatory on the pelvic limbs and were admitted to our veterinary hospitals to undergo anesthesia, cross sectional diagnostic imaging, and surgical decompression. Oral glyburide was given to each dog at a dose of $75 \mathrm{mcg} / \mathrm{kg}$. In 5 dogs, we measured blood glucose concentrations for 10 hours after a single oral dose. In 6 dogs, we measured serum glyburide and glucose concentrations for 24 hours and estimated pharmacokinetic parameters to estimate a suitable dose for use in a subsequent clinical trial in similarly affected dogs. Results. No detrimental effects of glyburide administration were detected in any participating dog. Peak serum concentrations of glyburide were attained at a mean of 13 hours after dosing, and mean apparent elimination half-life was approximately 7 hours. Observed mean maximum plasma concentration was $31 \mathrm{ng} / \mathrm{mL}$. At the glyburide dose administered there was no observable association between glyburide and glucose concentrations in blood. Discussion. Our data suggest that glyburide can be safely administered to dogs that are undergoing anesthesia, imaging and surgery for treatment of their acute spinal cord injury and can attain clinically-relevant serum concentrations without developing hazardous hypoglycemia. 
Serum glyburide concentrations achieved in this study suggest that a loading dose of 150 $\mathrm{mcg} / \mathrm{kg}$ followed by repeat doses of $75 \mathrm{mcg} / \mathrm{kg}$ at 8-hourly intervals would lead to serum glyburide concentrations of $25-50 \mathrm{ng} / \mathrm{mL}$ within an acceptably short enough period after oral administration to be appropriate for a clinical trial in canine spinal cord injury. 
1

2

3

4 Nick D Jeffery ${ }^{1}$, C. Elizabeth Boudreau ${ }^{1}$, Megan Konarik ${ }^{2}$, Travis Mays ${ }^{2}$, Virginia Fajt ${ }^{3}$

5

6

7

$8{ }^{1}$ Department of Small Animal Clinical Sciences, TAMU 4474, College of Veterinary Medicine,

9 Texas A\&M University, College Station, TX 77843, USA

10

11

12

13

14

15

16

17

18

19

20

\section{Pharmacokinetics and safety of oral glyburide in dogs with acute spinal cord injury}

(1)

5

6

(1)

10

$1{ }^{2}$ Veterinary Medical Diagnostic Laboratory, Texas A\&M University, PO Drawer 3040, College

12 Station TX 77841, USA

3

$4{ }^{3}$ Department of Veterinary Physiology \& Pharmacology, 4466 TAMU, Texas A\&M University, 5 College Station, TX 77843, USA

Corresponding author: Nick D Jeffery, njeffery@cvm.tamu.edu

9 
21 Abstract

22

Background. Glyburide (also known as glibenclamide) is effective in reducing the severity of tissue destruction and improving functional outcome after experimental spinal cord injury in rodents and so has promise as a therapy in humans. There are many important differences between spinal cord injury in experimental animals and in human clinical cases, making it difficult to introduce new therapies into clinical practice. Spinal cord injury is also common in pet dogs and requires new effective therapies, meaning that they can act as a translational model for the human condition while also deriving direct benefits from such research. In this study we investigated the pharmacokinetics and safety of glyburide in dogs with clinical spinal cord injury.

Methods. We recruited dogs that had incurred an acute thoracolumbar spinal cord injury within the previous 72 hours. These had become acutely non-ambulatory on the pelvic limbs and were admitted to our veterinary hospitals to undergo anesthesia, cross sectional diagnostic imaging, and surgical decompression. Oral glyburide was given to each dog at a dose of $75 \mathrm{mcg} / \mathrm{kg}$. In 5 dogs, we measured blood glucose concentrations for 10 hours after a single oral dose. In 6 dogs, we measured serum glyburide and glucose concentrations for 24 hours and estimated pharmacokinetic parameters to estimate a suitable dose for use in a subsequent clinical trial in similarly affected dogs.

Results. No detrimental effects of glyburide administration were detected in any participating dog. Peak serum concentrations of glyburide were attained at a mean of 13 hours after dosing, and mean apparent elimination half-life was approximately 7 hours. Observed mean maximum plasma concentration was $31 \mathrm{ng} / \mathrm{mL}$. At the glyburide dose administered there was no observable association between glyburide and glucose concentrations in blood.

Discussion. Our data suggest that glyburide can be safely administered to dogs that are undergoing anesthesia, imaging and surgery for treatment of their acute spinal cord injury and can attain clinically-relevant serum concentrations without developing hazardous hypoglycemia. Serum glyburide concentrations achieved in this study suggest that a loading dose of $150 \mathrm{mcg} / \mathrm{kg}$ 
52 followed by repeat doses of $75 \mathrm{mcg} / \mathrm{kg}$ at 8 -hourly intervals would lead to serum glyburide

53 concentrations of $25-50 \mathrm{ng} / \mathrm{mL}$ within an acceptably short enough period after oral

54 administration to be appropriate for a clinical trial in canine spinal cord injury.

\section{Introduction}

Despite extensive research on the mechanisms mediating the transition from traumatic spinal cord injury to tissue destruction, an unequivocally-accepted medical intervention for reduction in tissue destruction has not been introduced into clinical medicine. This failure is especially surprising bearing in mind the large number of interventions that have been effective in experimental rodent models (Kwon et al., 2011; Tetzlaff et al., 2011). For that reason, it appears that, rather than a lack of discovery of putative interventions, the roadblock to introduction of new therapies lies in translating new therapies from laboratory to clinic.

There is a 'large translational gap' between rodent experiments and human clinical practice meaning that testing in additional models is required to bolster confidence in taking forward specific interventions to human clinical trials (Kwon et al., 2015). There is a role for many models to achieve this aim: for instance, laboratory models of spinal cord injury in primates are important in investigation of strategies to improve hand use (Salegio et al., 2016) and pig models are useful for making measurements of intradural pressure following experimental spinal cord injury (Streijer et al., 2017). However, an important difference between laboratory models and human spinal cord injury is the heterogeneity of clinical patients; notably they vary in genetics, age, comorbidities and time delay between injury and access to therapy. Such heterogeneity introduces several sources of variation into outcome measures and implies that apparent effectiveness of an intervention in the laboratory may not be apparent when applied in clinical patients. It is difficult to model this disparity using laboratory animals but spinal-injured veterinary patients mimic both lesion and treatment of human spinal cord injury. Pet dogs show considerable heterogeneity (similar to human patients), commonly incur spinal cord injury in the course of their everyday lives and undergo similar diagnostic and therapeutic interventions as human patients, with similar limitations in their recovery rates (Moore et al., 2017). Therefore, there are many benefits to investigating putative therapies for spinal cord injury in canine 
83

84

85

86

87

88

veterinary patients. In addition, from a veterinary perspective, it is also necessary to identify new therapies for the dogs that incur such injuries.

Glyburide (also known as glibenclamide) has been widely studied in treatment of many CNS lesions in the laboratory, including stroke (Simard et al., 2006), traumatic brain injury (Simard et al., 2009) and spinal cord injury (Simard et al., 2007; 2012; Popovich et al., 2012). Glyburide's mechanism of action in CNS injury is via blockade of the Sur1-Trpm4 channel that is highly upregulated after CNS injury, particularly in blood vessels (Simard et al., 2007; Gerzanich et al., 2009). The Sur1-Trpm4 channel allows ingress of cations into affected cells, resulting in cytotoxic edema and cell lysis (Simard et al., 2010). Glyburide binds to Sur1 at very low concentrations (10-100nM; Aguilar-Bryan et al., 1990), especially at the low $\mathrm{pH}$ found in ischemic tissue (Simard et al., 2008) and its effects compare well with other drugs that might aid in preserving damaged spinal cord after insult when tested in rodent models (Hosier et al., 2015). Furthermore, it is an attractive candidate for translation because its pharmacological effects are well-known, a dose range to achieve therapeutic levels has been calculated for human clinical trials (Sheth et al., 2016a), the drug is in widespread use (in humans) for treatment of type II diabetes (Rendell, 2004) and it is widely available as an inexpensive oral preparation. Pancreatic beta cells also express Sur1 receptors (Panten et al., 1996) meaning that the drug effects on spinal cord endothelial (and other) cells are mediated via the same mechanism as that on blood glucose. Therefore, the main safety concern with use of this drug for spinal cord injury is hypoglycemia.

The safety of oral glyburide in healthy dogs has been evaluated previously (Guan et al., 2014; Liu et al., 2014; http://products.sanofi.ca/en/diabeta.pdf). In this study we wished to extend these assessments into a cohort of dogs that had spinal cord injuries for which they were undergoing investigation and surgery because these other interventions and the lesion itself may alter blood drug concentrations or risk of adverse effects. For instance, drug absorption can be compromised by the reduction in gut motility associated with anesthesia (Torjman et al., 2005) or spinal cord injury itself (Cruz-Antonio et al., 2006; 2012). Specifically, our objectives were to evaluate the effects on blood glucose of glyburide and to assess drug disposition, in order to make recommendations for dose regimens for a subsequent clinical trial in dogs with similar injuries. 


\section{Materials \& Methods}

116 We recruited pet dogs with acute, naturally-occurring spinal cord injury. Dogs affected in this

117 way routinely undergo general anesthesia, cross-sectional imaging, decompressive spinal surgery, and they receive intravenous fluid infusions, any of which might affect drug disposition or the effects of glyburide on blood glucose. Animals were treated in this study as they would be clinically, for instance, intravenous fluid was given according to each individual's requirements as determined by the attending anesthetist, because we were interested in mimicking future clinical application of glyburide. There were several phases to this study: i) testing whether oral glyburide caused hypoglycemia in the target patient population; ii) determining concentrations of glyburide over the 24-hour period following a single oral dose of glyburide administered just before induction of anesthesia for imaging and surgery; and, iii) estimating a dose regimen that would achieve a plasma concentration of glyburide between 25 and $50 \mathrm{ng} / \mathrm{mL}$ for at least 72 hours (Simard et al., 2008; Sheth et al., 2016).

\section{Animals}

Dogs included in this study presented for treatment of acute spinal cord injury to the small animal hospital of the Colleges of Veterinary Medicine at Iowa State University or Texas A\&M University, and study protocols were approved by the relevant Institutional Animal Care and Use Committees (Iowa State, log number: 1-16-8148-K; Texas A\&M, IACUC 2016-0324 CA, reference number 044949). For inclusion, dogs had to have become acutely non-ambulatory with suspected intervertebral disc herniation occurring less than 72 hours previously and be ready to undergo general anesthesia and surgery (i.e. fasted); those that were thought likely to have lesions other than intervertebral disc herniation, or that were not likely to undergo imaging and surgery, were not invited to take part. Dogs in either of these categories that were initially included (i.e. given the drug) were subsequently excluded from further analysis. Dogs diagnosed with hyperadrenocorticism or diabetes mellitus were excluded.

\section{Materials}

143 For glyburide dosing we obtained commercially available generic glyburide tablets of $1.5 \mathrm{mg}$ 144 (Teva) and $1.25 \mathrm{mg}$ (Heritage) each. Blood glucose was measured using a commercially- 
145 available glucometer and test strips designed for diabetic monitoring in dogs (AlphaTrak2,

146 Abbott Laboratories, Abbott Park, IL, USA); the performance of this testing method against

147 reference standards has been previously published (Cohen et al., 2009).

148

149 For measurement of glyburide concentration in blood samples, control normal canine plasma 150 was obtained from Equitech-Bio, Inc., Kerrville, TX, USA (\#SCAPE35-0100). Glyburide (USP

151 Reference, \#1295505) and Glipizide (G117) reference materials were obtained from Sigma, St.

152 Louis, MO, USA. PBS Buffer, $\mathrm{pH}$ 7.0, was made using $8 \mathrm{~g} \mathrm{NaCl}, 0.2 \mathrm{~g} \mathrm{KCl}, 1.44 \mathrm{~g} \mathrm{Na}_{2} \mathrm{HPO}_{4}$, 153 and $0.24 \mathrm{~g} \mathrm{KH}_{2} \mathrm{PO}_{4}$ dissolved in $1 \mathrm{~L}$ reverse-osmosis deionized (RO-DI) water, $\mathrm{pH}$ adjusted to 1547.0 with a $6 \mathrm{~N} \mathrm{HCl}$ solution, sourced in-house. All chemicals and reagents were ACS grade and 155 obtained from VWR Scientific, Randor, PA, USA.

Methods

159 1. Safety study

160 Five dogs that were paraplegic and underwent imaging and surgery under general anesthesia were included; in this part of the study there were no dog weight restrictions applied. After receiving informed owner consent, each dog received $75 \mathrm{mcg} / \mathrm{kg}$ glyburide orally; the dose was chosen based on previous safety studies in dogs (http://products.sanofi.ca/en/diabeta.pdf) and the dosages given in similar human clinical trials (https://www.clinicaltrials.gov/ct2/show/NCT02524379?term=glyburide + spinal + cord\&rank=1; Sheth et al., 2014). Partial tablets were used as appropriate for the weight of the dog. At hourly intervals from 0 to 4 hours, and 2-hourly intervals from 4 to 10 hours after drug administration, a single drop of blood was withdrawn by needle puncture of a peripheral vein and tested for blood glucose concentration using a blood glucose test strip (AlphaTrak2). If a value of less than 50 $\mathrm{mg} / \mathrm{dL}$ was recorded then that individual received intravenous glucose as required to normalize the glucose to within the laboratory reference interval $(76-119 \mathrm{mg} / \mathrm{dL})$. occurred at any time (with direction to pay particular attention to signs of ataxia, disorientation, or other potential signs of hypoglycemia), and notes were made of any need for supplementary 
176 glucose administration. [It should be noted that blood glucose is not routinely monitored in dogs

177 undergoing anesthesia and surgery.]

178

179

180

\section{Pharmacokinetic study}

Six dogs weighing more than $5 \mathrm{~kg}$ and undergoing imaging and surgery for treatment of acute

181

182

183

184

185

186

187

188

189

190

191

192

193

194

195

196

197

198

199

200

201

202

203

204

205

intervertebral disc herniation resulting in non-ambulatory paraparesis or paraplegia were included in this part of the study. As before, after obtaining informed consent, each dog was given $75 \mathrm{mcg} / \mathrm{kg}$ glyburide orally as a tablet or broken tablet. Blood samples $(1 \mathrm{~mL})$ were obtained from a peripheral vein via a preplaced long catheter to measure glyburide concentration and evaluate blood glucose concentrations at 1, 2, 3, 4, 5, 6, 8, 10, 12, 14, 16, 20, and 24 hours after drug administration. Before each blood sample was obtained an aliquot of $5 \mathrm{~mL}$ blood was withdrawn through the catheter into a heparinized syringe, retained, and then injected back into the dog after the test sample had been obtained. Each $1 \mathrm{~mL}$ blood sample was tested for blood glucose concentration using a glucose test strip (AlphaTrak2). In this study (because of different IACUC recommendations at different institutions, which also altered our dog weight inclusion criteria), if a value of less than $60 \mathrm{mg} / \mathrm{dL}$ was recorded, then intravenous glucose was administered as required to normalize the glucose to within the laboratory reference interval (76 $-119 \mathrm{mg} / \mathrm{dL})$. The remainder of the sample was placed into a heparinized plastic blood collection tube. This second aliquot was centrifuged at $2500 \mathrm{G}$ for 15 minutes and then the plasma was decanted into microcentrifuge tubes and frozen at $-80{ }^{\circ} \mathrm{C}$ until analyzed.

When all plasma samples had been collected from all six dogs, glyburide concentration was measured by liquid chromatography / tandem mass spectrometry (LC/MC/MC). Using $300 \mu \mathrm{L}$ negative canine plasma, the calibration curve was spiked accordingly, with calibrator concentrations of $1,5,10,20,50,100,250$, and $500 \mathrm{ng} / \mathrm{mL}$. Sample aliquots of $300 \mu \mathrm{L}$ were used, and $50 \mu \mathrm{L}$ glipizide were added to all samples as the internal standard. Each sample was diluted with $1700 \mu \mathrm{L}$ PBS Buffer, $\mathrm{pH}$ 7.0, making a final volume of $2 \mathrm{~mL}$. Samples were vortexed and allowed to rest at room temperature for approximately 5 minutes before solid phase extraction (SPE). 
206 Samples were extracted by SPE using the SPEWare CEREX48 Processor (SPEWare Corp.,

207 Baldwin Park, CA, USA). Water Wettable Polymer (WWP) SPE cartridges (SPEWare \#12-

208 170418) were used, conditioned with $1 \mathrm{~mL}$ methanol, $1 \mathrm{~mL}$ RO-DI water, and $1 \mathrm{~mL}$ PBS Buffer,

$209 \mathrm{pH}$ 7.0. Samples were added to cartridges and allowed to filter through at $1-2 \mathrm{~mL} / \mathrm{min}$.

210 Cartridges were washed with $1 \mathrm{~mL}$ RO-DI water and dried at full pressure (80 psi) for

211 approximately 10 minutes. Samples were eluted with $1 \mathrm{~mL}$ methanol and dried to a residue under

212 nitrogen at $40^{\circ} \mathrm{C}$. They were reconstituted in $100 \mu \mathrm{L}$ of a 95:5 reagent grade water:acetonitrile

213 solution before analysis by LC/MS/MS.

214

215

216

Samples were analyzed using a Thermo TSQ Endura LC/MS/MS (Thermo Instruments, San

217

Jose, CA, USA) system. The analytes were separated using an Agilent Eclipse Plus C18 2.1 x 50

218

mm, $1.8 \mu \mathrm{m}$ column (\#959757-902, Agilent Technologies, Santa Clara, CA, USA). The mobile

219

phases were composed of $0.1 \%$ formic acid in water (Mobile Phase A) and $0.1 \%$ Formic acid in

220

acetonitrile (Mobile Phase B). The gradient began at 25\%B and increased to $95 \% \mathrm{~B}$ to 3.0

221

minutes with a flow of $250 \mu \mathrm{L} / \mathrm{min}$, then increased flow to $500 \mu \mathrm{L} / \mathrm{min}$ for $0.7 \mathrm{~min}$. The

222

LC/MS/MS used a HESI ion source in positive ion mode and a mass resolution of 0.7 on both

223

224

quadrupoles. Supplementary Table 1 summarizes the mass spectrometer conditions.

225

3. Pharmacokinetic calculations

225

Non-compartment and compartmental analyses were performed to provide estimates of various pharmacokinetic parameters. Non-compartmental analysis makes no assumption about the underlying model of disposition behavior, so it is useful as a descriptive approach. However, compartmental models can be more useful for making predictions and for explaining observed drug disposition characteristics. Therefore, in this study, we performed both to provide both approaches to estimating pharmacokinetic parameters.

231

Non-compartmental analysis was performed to estimate various pharmacokinetic parameters of glyburide for each individual animal. The following parameters were estimated: time of observed peak plasma drug concentration $\left(\mathrm{T}_{\max }\right)$, observed peak drug concentration $\left(\mathrm{C}_{\max }\right)$, apparent elimination half-life $\left(\mathrm{t}_{1 / 2}\right.$, calculated as $\ln (2) / \lambda_{z}, \lambda_{z}$ being the first-order rate constant associated with the terminal portion of the time-concentration curve as estimated by linear regression of 
237 time versus log concentration ( $\mathrm{AUC}_{0 \text {-last }}$, calculated by the linear trapezoidal rule), area under the 238 time-concentration curve from time zero extrapolated to infinity $\left(\mathrm{AUC}_{0 \text {-inf }}\right.$, calculated by adding

239 the last observed concentration divided by $\lambda_{\mathrm{z}}$ to the $\mathrm{AUC}_{0 \text {-last }}$ ), area under the moment curve from 240 time zero to last observed concentration ( $\mathrm{AUMC}_{0 \text {-last}}$ ), area under the moment curve from time 241 zero extrapolated to infinity $\left(\mathrm{AUMC}_{0 \text {-inf }}\right)$, mean resident time estimated using time zero to last 242 observed concentrations ( $\mathrm{MRT}_{0 \text {-last }}$, calculated as $\mathrm{AUMC}_{0 \text {-last }} / \mathrm{AUC}_{0 \text {-last }}$ ), and mean residence 243 time estimated using time zero to infinity ( $\mathrm{MRT}_{0 \text {-inf }}$, calculated as $\mathrm{AUMC}_{0 \text {-inf }} / \mathrm{AUC}_{0 \text {-inf }}$ ).

245 Compartmental modeling was also attempted to estimate the pharmacokinetic parameters in 246 plasma for each individual animal. One- and two-compartment models were attempted, and 247 Akaike's Information Criterion and visual assessment of observed versus predicted values were 248 used to select the best fit. The following parameters were estimated for each animal: observed 249 time of peak plasma drug concentration $\left(\mathrm{T}_{\max }\right)$, observed peak drug concentration $\left(\mathrm{C}_{\max }\right)$, 250 apparent absorption and elimination rate $(\mathrm{K})$, apparent absorption and elimination half-life $\left(\mathrm{t}_{1 / 2}\right)$, 251 area under the time-concentration curve (AUC), time from drug administration to onset of 252 absorption $\left(\mathrm{T}_{\mathrm{lag}}\right)$, volume of distribution corrected for bioavailability (V/F), and clearance 253 corrected for bioavailability $(\mathrm{Cl} / \mathrm{F})$. All analyses were performed in industry-standard 254 pharmacokinetic software (WinNonLin 7.0.0.2535, Pharsight, Princeton, NJ, USA). 255

Finally, the available data were used to estimate the drug dosage regimen needed to achieve blood concentrations of glyburide between 25 and $50 \mathrm{ng} / \mathrm{mL}$ for at least 72 hours. We stipulated that, for convenience of subsequent clinical use of this drug, dosing could not be more frequent than every 8 hours. Nonparametric superpositioning in Phoenix WinNonLin was used to create a graph of the mean estimated concentrations of glyburide for 72 hours of a dosing regimen consisting of a loading dose of $150 \mathrm{mcg} / \mathrm{kg}$, followed by $75 \mathrm{mcg} / \mathrm{kg}$ every 8 hours in Dogs 2-5 (the observed data from these dogs had reasonable terminal slopes from which to calculate the 263 elimination rate).

\section{Results}


268

269

270

271

272

273

274

275

276

277

278

279

280

281

282

283

284

285

286

287

288

289

290

291

292

293

294

295

296

297

\section{Safety study}

Five dogs were included: 5 year-old Bichon Frise mixed breed (10.2 kg), 9 year-old Dachshund (8.1kg), 11 year-old Cardigan Corgi (14kg), 6 year-old Dachshund (6.8kg), 6 year-old Dachshund (4.2kg). An initially-enrolled Dobermann was withdrawn from the study at 3 hours because a diagnosis of spinal neoplasia was made.

No adverse effects were noted in any dog receiving glyburide. Blood glucose concentrations of less than $50 \mathrm{mg} / \mathrm{dL}$ were not observed in any dog, although this exact value was reached in one $\operatorname{dog} 2$ hours after receiving the $75 \mathrm{mcg} / \mathrm{kg}$ oral dose of glyburide.

Overall, blood glucose concentrations appeared largely unaffected by administration of this dose of glyburide to dogs in this study and remained more-or-less constant throughout the 10-hour period of observation (Fig. 1). Blood concentrations for each dog are shown in Supplementary Table 2 .

\section{Pharmacokinetic study}

Six dogs were included in this part of the study: 6 year-old Dachshund $(6.7 \mathrm{~kg}), 4$ year-old German Short-haired Pointer (20.3kg), 4 year-old French Bulldog (9.2kg), 10 year-old Shi Tzu (7.8kg), 5 year-old Dachshund (7.8kg), 4 year-old Dachshund (7.6kg). Mean peak glyburide concentration was reached at 13 hours after oral administration, and peak concentrations exceeded $25 \mathrm{ng} / \mathrm{mL}$ in four of the six dogs but did not reach this concentration in the remaining two (Dog 1 and Dog 6). Values exceeding 25ng/mL were detected once each in Dog 2 and Dog 4, twice in Dog 5 and five times in Dog 3 (Supplementary Table 3).

Serum concentration data and pharmacokinetic parameter estimates suggest that Dog 6 is an outlier: $\mathrm{T}_{\max }$ was at the last observed time point for this dog (see Table 1), and the plasma concentrations for this dog were much lower than the other dogs at all time points, resulting in a skewing of mean observed serum concentrations (Fig. 2). In addition, compartmental pharmacokinetic analysis was not possible for Dog 6 with any of the attempted models. In the non-compartmental analysis, two dogs (Dog 1 and Dog 2) required higher than desirable 
298

299

300

301

302

303

304

305

306

307

308

309

310

311

312

313

314

315

316

317

318

319

320

321

322

323

324

325

326

327

328

extrapolation percentages to calculate AUC: both were greater than $20 \%$, the recommended maximal extrapolation percentage.

\section{Relationship between glyburide and glucose concentrations in pharmacokinetic study} Blood glucose concentration ranged from 50 to $324 \mathrm{mg} / \mathrm{dL}$ (Supplementary Table 4). In one dog the blood glucose concentration reached $50 \mathrm{mg} / \mathrm{dL}$ at 4 hours after drug administration, which was lower than the pre-defined intervention level of $60 \mathrm{mg} / \mathrm{dL}$ for this study, and intravenous glucose supplementation was given; subsequent recordings of blood glucose concentrations were excluded in the analysis of glucose versus glyburide concentration for this dog. Linear regression analysis and plot (Fig. 3) of the relationship between drug and glucose concentration in our whole study population suggested there was not a significant association between these variables at this glyburide dosage $\left(\mathrm{R}^{2}=0.008 ; \mathrm{P}=0.464\right)$.

Superpositioning of observed concentrations in Dogs 2-5 using a loading dose of $150 \mathrm{mcg} / \mathrm{kg}$ glyburide followed by 8 -hourly dosing at $75 \mathrm{mcg} / \mathrm{kg}$ produced an average plasma level of above $25 \mathrm{ng} / \mathrm{mL}$, but below $50 \mathrm{ng} / \mathrm{mL}$, for most of a simulated 72-hour treatment period (Fig. 4).

\section{Discussion}

We show here that our conservatively-selected glyburide dosage of $75 \mathrm{mcg} / \mathrm{kg}$ achieved the target blood level of between 25 and $50 \mathrm{ng} / \mathrm{mL}$ in only four of six dogs in our pharmacokinetic study, and the periods for which this was achieved were brief. On the other hand, this dose did not cause unsafe reductions in blood glucose concentrations in any of the dogs. In two dogs there was a reduction in blood glucose to $<60 \mathrm{mg} / \mathrm{dL}$ but neither of these animals exhibited any illeffects, although they were anesthetized at the time. It seems likely that these periods of hypoglycemia were not the result of glyburide itself since there appeared to be no relationship between glyburide and glucose blood concentrations in these dogs at this dosage, suggesting that glyburide can be safely administered at this, or higher, doses, as previously reported (http://products.sanofi.ca/en/diabeta.pdf). All 11 dogs recruited to this study uneventfully recovered the ability to walk after surgery. 
329 In a clinical trial evaluating glyburide for acute spinal cord injury, because it is a rapidly

330 evolving condition, there is a need to achieve effective blood concentrations as rapidly as

331 possible and maintain these for at least 72 hours (in line with other clinical uses of this drug;

332 Sheth et al., 2016a; 2016b). We estimate that a safe and effective dose regimen would be a

333 loading dose of $150 \mathrm{mcg} / \mathrm{kg}$, followed by $75 \mathrm{ng} / \mathrm{kg}$ every 8 hours. By extrapolation from the data

334 we have collected in this study such a regimen is expected to lead to therapeutic concentrations

335 (>25ng/mL; see Simard et al., 2008) 2-3 hours after dosing and for up to for 72 hours. The

336 loading dose is proposed as a means to ensure that the drug attains a therapeutic concentration as

337 soon as possible after oral dosing. The assumption in treatment of spinal cord injury is that rapid

338 treatment is essential for effective neuroprotection. Although intravenous preparations (see Sheth

339 et al., 2014) may be preferable for this reason, in veterinary medicine much of the delay between

340 onset of injury and initiation of treatment is caused by delay in owner recognition of the

341 condition, travel to a specialist clinic, triage and obtaining owner consent for treatment, which

342 cannot easily be eliminated. Fortunately, although the stimulus for the secondary injury

343 mechanisms of spinal cord injury is the moment of impact, it can take hours to days for the

344 tissue-destructive mechanisms to become fully up-regulated (Crowe et al., 1997). Indeed, Sur1,

345 the target of glyburide, becomes increasingly widely expressed during the first 24 hours after

346 experimental spinal cord injury (Simard et al., 2007), suggesting that, although earlier

347 administration is likely to be superior, attaining appropriate plasma drug concentrations within 2-

3483 hours after examination at a veterinary clinic may still be efficacious.

An important aspect of this current study is that we used a sample of dogs similar to those that

351

352

353

354

355

356

357

358

359 will be targeted by this therapy in a clinical trial. Although such cases will produce more heterogeneous pharmacokinetic data than normal, conscious, laboratory animals, it is important to assess drug disposition and adverse effects on blood glucose that could possibly occur in this category of veterinary patient. Specifically, dogs undergoing anesthesia for imaging and surgery are routinely administered intravenous fluids that by expanding the circulating volume may affect drug concentration and, in addition, may receive other drugs including antibiotics, analgesics and gaseous anesthetic agents, all of which may alter glyburide concentration in blood or its effects on blood glucose. Our study shows that despite these factors the dose of $75 \mathrm{mcg} / \mathrm{kg}$ can achieve sufficiently high concentrations to affect function of the Sur1 channel (see Simard et 
360 al., 2008) and has no detectable adverse effects. Our data also imply that the dose will need to be

361 scaled-up to achieve rapid and maintained plasma concentrations adequate to achieve the desired 362 clinical benefit.

363

364 Conclusions

365 This study shows that glyburide given orally at $75 \mathrm{mcg} / \mathrm{kg}$ only just achieves blood

366 concentrations of the drug appropriate for targeting function of the Sur1 channel in cells in the

367 injured spinal cord but does not appear to adversely affect blood glucose concentrations. To

368 achieve rapid and appropriate concentrations of glyburide we suggest using an initial loading

369 dose of $150 \mathrm{mcg} / \mathrm{kg}$ followed by repeat dosing at $75 \mathrm{mcg} / \mathrm{kg}$ every 8 hours.

370

371

372

373

374

375

376

377

378

379

380

381

382

383

384

385

386

387

388

389

390

\section{Acknowledgement}

We thank the 'Dogs Helping Dogs' laboratory at Texas A\&M University for access to their equipment for blood sample processing.

\section{Figure legends}

Figure 1: Relationship between blood glucose concentration and time after oral administration of $75 \mathrm{mcg} / \mathrm{kg}$ glyburide at time 0 ; bars indicate standard error of the mean (s.e.m.).

Figure 2: Relationship between serum glyburide concentration and time after oral administration of $75 \mathrm{mcg} / \mathrm{kg}$ glyburide at time 0; bars indicate s.e.m. Dashed horizontal line indicates minimum desired therapeutic concentration (see text).

Figure 3: Relationship between blood glucose and serum glyburide concentrations. The line indicates linear regression line of best fit (dotted lines indicate 95\% confidence intervals) implying that these variables are not significantly associated (for glyburide concentrations in this range); $\mathrm{R}^{2}=0.008 ; \mathrm{P}=0.464$. 
391

392 393

394

395

396

397

398

399

400

401

402

403

404

405

406

407

408

409

410

411

412

413

414

415

416

417

418

419

420

421

Figure 4: Predicted glyburide concentrations based on superpositioning of observed concentrations in individual dogs. Shaded area represents one standard deviation from mean.

\section{References}

Aguilar-Bryan L, Nelson DA, Vu QA, Humphrey MB, Boyd AE 3 ${ }^{\text {rd }}$. 1990. Photoaffinity labeling and partial purification of the beta cell sulfonylurea receptor using a novel, biologically active glyburide analog. Journal of Biological Chemistry 265: 8218-8224.

Cohen TA, Nelson RW, Kass PH, Christopher MM, Feldman EC. 2009. Evaluation of six portable blood glucose meters for measuring blood glucose concentration in dogs. Journal of the American Veterinary Medical Association 235: 276-80.

Crowe MJ, Bresnahan JC, Shuman SL, Masters JN, Beattie MS. 1997. Apoptosis and delayed degeneration after spinal cord injury in rats and monkeys. Nature Medicine 3: 73-6.

Cruz-Antonio L, Flores-Murrieta FJ, García-Löpez P, Guízar-Sahagún G, Castañeda-Hernández G. 2006. Understanding drug disposition alterations induced by acute spinal cord injury: role of injury level and route of administration for agents submitted to extensive liver metabolism. Journal of Neurotrauma 23: 75-85.

Cruz-Antonio L, Arauz J, Franco-Bourland RE, Guízar-Sahagún G, Castañeda-Hernández G. 2012. Contrasting effects of cord injury on intravenous and oral pharmacokinetics of diclofenac: a drug with intermediate hepatic extraction. Spinal Cord 50: 632-5.

Gerzanich V, Woo SK, Vennekens R, Tsymbalyuk O, Ivanova S, Ivanov A, Geng Z, Chen Z, Nilius B, Flockerzi V, Freichel M, Simard JM. 2009. De novo expression of Trpm4 initiates secondary hemorrhage in spinal cord injury. Nature Medicine 15:185-191. 
422 Guan J, Han J, Zhang D, Chu C, Liu H, Sun J, He Z, Zhang T. 2014. Increased dissolution

423 rate and oral bioavailability of hydrophobic drug glyburide tablets produced using supercritical

$424 \mathrm{CO}_{2}$ silica dispersion technology. European Journal of Pharmaceutics and Biopharmaceutics 86:

$425 \quad 376-82$

426

427 Hosier H, Peterson D, Tsymbalyuk O, Keledjian K, Smith BR, Ivanova S, Gerzanich V, 428 Popovich PG, Simard JM. 2015. A Direct Comparison of Three Clinically Relevant Treatments 429 in a Rat Model of Cervical Spinal Cord Injury. Journal of Neurotrauma 32: 1633-44.

430

431 http://products.sanofi.ca/en/diabeta.pdf

432

433 Kwon BK, Streijger F, Hill CE, Anderson AJ, Bacon M, Beattie MS, Blesch A, Bradbury EJ, 434 Brown A, Bresnahan JC, Case CC, Colburn RW, David S, Fawcett JW, Ferguson AR, Fischer I, 435 Floyd CL, Gensel JC, Houle JD, Jakeman LB, Jeffery ND, Jones LA, Kleitman N, Kocsis J, Lu 436 P, Magnuson DS, Marsala M, Moore SW, Mothe AJ, Oudega M, Plant GW, Rabchevsky AS, 437 Schwab JM, Silver J, Steward O, Xu XM, Guest JD, Tetzlaff W. 2015. Large animal and primate 438 models of spinal cord injury for the testing of novel therapies. Experimental Neurology 269: $439 \quad 154-68$.

440

Kwon BK, Okon E, Hillyer J, Mann C, Baptiste D, Weaver LC, Fehlings MG, Tezlaff W. 2011. A systematic review of non-invasive pharmacologic neuroprotective treatments for acute spinal 443 cord injury. Journal of Neurotrauma 28: 1545-88.

Liu H, Shang K, Liu W, Leng D, Li R, Kong Y, Zhang T. 2014. Improved oral bioavailability of 446 glyburide by a self-nanoemulsifying drug delivery system. Journal of Microencapsulation 31: 277-83.

Moore SA, Granger N, Olby NJ, Spitzbarth I, Jeffery ND, Tipold A, Nout-Lomas YS, da Costa 450 RC, Stein VM, Noble-Haeusslein LJ, Blight AR, Grossman RG, Basso DM, Levine JM. 2017.

451 Targeting translational successes through CANSORT-SCI: Using pet dogs to identify effective 452 treatments for spinal cord injury. Journal of Neurotrauma 34: 2007-18. 
454 Panten U, Schwanstecher M, Schwanstecher C. 1996. Sulfonylurea receptors and mechanism of 455 sulfonylurea action. Experimental and Clinical Endocrinology and Diabetes 104: 1-9.

456

457 Popovich PG, Lemeshow S, Gensel JC, Tovar CA. 2012. Independent evaluation of the effects 458 of glibenclamide on reducing progressive hemorrhagic necrosis after cervical spinal cord injury. 459 Experimental Neurology 233: 615-22.

460

461

Rendell M. 2004. The role of sulphonylureas in the management of type 2 diabetes mellitus.

462 Drugs 64: 1339-58.

463

464

Salegio EA, Bresnahan JC, Sparrey CJ, Camisa W, Fischer J, Leasure J, Buckley J, Nout-Lomas 465 YS, Rosenzweig ES, Moseanko R, Strand S, Hawbecker S, Lemoy MJ, Haefeli J, Ma X, Nielson 466 JL, Edgerton VR, Ferguson AR, Tuszynski MH, Beattie MS. 2016. A Unilateral Cervical Spinal Cord Contusion Injury Model in Non-human Primates (Macaca mulatta). Journal 468 of Neurotrauma 33: 439-59.

469

470

Sheth KN, Elm JJ, Beslow LA, Sze GK, Kimberly WT. 2016a. Glyburide Advantage in Malignant Edema and Stroke (GAMES-RP) Trial: Rationale and Design.

472 Neurocritical Care 24: 132-9.

473

474

Sheth KN, Elm JJ, Molyneaux BJ, Hinson H, Beslow LA, Sze GK, Ostwaldt AC, Del Zoppo GJ, 475 Simard JM, Jacobson S, Kimberly WT. 2016b. Safety and efficacy of intravenous glyburide on brain swelling after large hemispheric infarction (GAMES-RP): a randomised, double-blind, placebo-controlled phase 2 trial. Lancet Neurology 15: 1160-9.

Sheth KN, Kimberly WT, Elm JJ, Kent TA, Mandava P, Yoo AJ, Thomalla G, Campbell B, 480 Donnan GA, Davis SM, Albers GW, Jacobson S, Simard JM, Stern BJ. 2014. Pilot study of 481 intravenous glyburide in patients with a large ischemic stroke. Stroke 45: 281-3. 
483 Simard J.M., Kilbourne M., Tsymbalyuk O., Tosun C., Caridi J., Ivanova S., Keledjian K., 484 Bochicchio G., Gerzanich V. 2009. Key role of sulfonylurea receptor 1 in progressive secondary 485 hemorrhage after brain contusion. Journal of Neurotrauma 26: 2257-2267.

486

487 Simard J.M., Tsymbalyuk O., Keledjian K., Ivanov A., Ivanova S., Gerzanich V. 2012.

488 Comparative effects of glibenclamide and riluzole in a rat model of severe cervical spinal cord 489 injury. Experimental Neurology 233: 566-574.

490

491 Simard JM, Chen M, Tarasov KV, Bhatta S, Ivanova S, Melnitchenko L, Tsymbalyuk N, West 492 GA, Gerzanich V. 2006. Newly expressed SUR1-regulated NC(Ca-ATP) channel mediates 493 cerebral edema after ischemic stroke. Nature Medicine 12: 433-440.

494

495

Simard JM, Kahle KT, Gerzanich V. 2010. Molecular mechanisms of microvascular failure in 496 central nervous system injury-synergistic roles of NKCC1 and SUR1/TRPM4. Journal of

497 Neurosurgery 113: 622-9.

498

Simard JM, Tsymbalyuk O, Ivanov A, Ivanova S, Bhatta S, Geng Z, Woo SK, Gerzanich V.

500 2007. Endothelial sulfonylurea receptor 1- regulated NC(Ca-ATP) channels mediate progressive 501 hemorrhagic necrosis following spinal cord injury. Journal of Clinical Investigation 117: 21055022113.

503

504 Simard JM, Woo SK, Bhatta S, Gerzanich V. 2008. Drugs acting on SUR1 to treat CNS 505 ischemia and trauma. Current Opinion in Pharmacology 8: 42-9.

506

507 Streijger F, So K, Manouchehri N, Tigchelaar S, Lee JHT, Okon EB, Shortt K, Kim SE, McInnes 508 K, Cripton P, Kwon BK. 2017. Changes in Pressure, Hemodynamics, and Metabolism within 509 the Spinal Cord during the First 7 Days after Injury Using a Porcine Model. Journal of 510 Neurotrauma 34: 3336-3350 
512 Tetzlaff W, Okon EB, Karimi-Abdolrezaee S, Hill CE, Sparling JS, Plemel JR, Plunet WT, Tsai

513 EC, Baptiste D, Smithson LJ, Kawaja MD, Fehlings MG, Kwon BK. 2011. A systematic review

514 of cellular transplantation therapies for spinal cord injury. Journal of Neurotrauma 28: 1611-82.

515

516 Torjman MC, Joseph JI, Munsick C, Morishita M, Grunwald Z. 2005. Effects of isoflurane on

517 gastrointestinal motility after brief exposure in rats. International Journal of Pharmacology 294:

$518 \quad 65-71$.

519 


\section{Figure 1 (on next page)}

Relationship between blood glucose concentration and time after oral administration of $75 \mathrm{mcg} / \mathrm{kg}$ glyburide at time 0; bars indicate standard error of the mean (s.e.m.). 


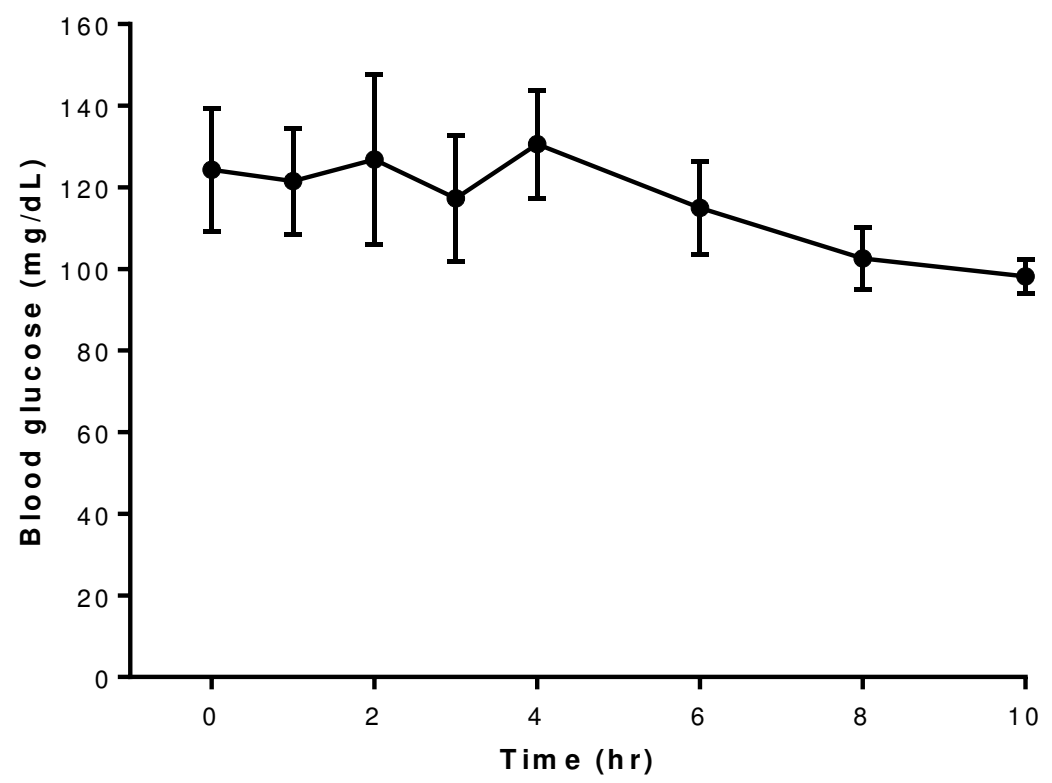




\section{Figure 2 (on next page)}

Relationship between serum glyburide concentration and time after oral administration of $75 \mathrm{mcg} / \mathrm{kg}$ glyburide at time 0 ; bars indicate s.e.m.

Dashed horizontal line indicates desired minimum therapeutic concentration (see text). 


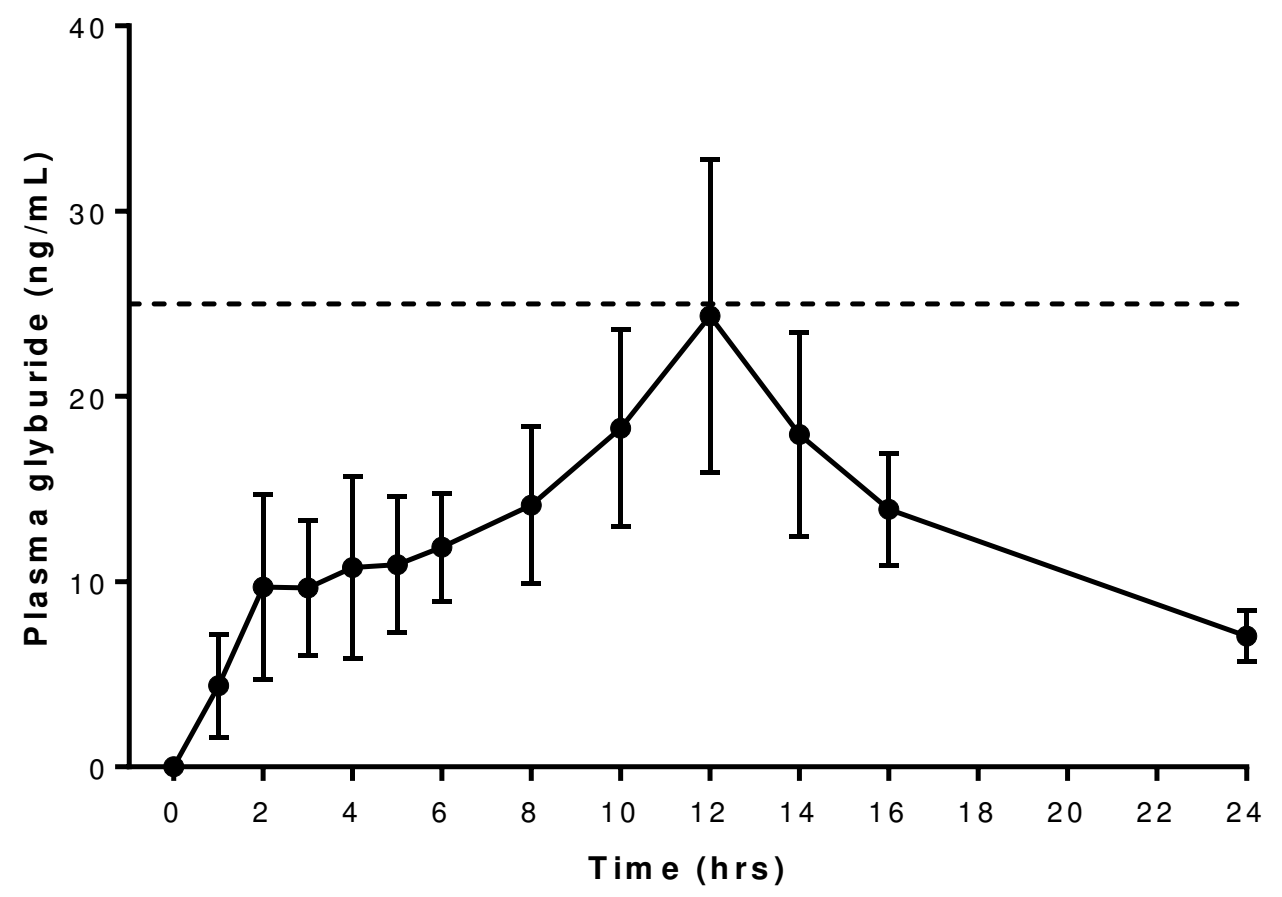




\section{Figure 3 (on next page)}

Relationship between blood glucose and serum glyburide concentrations.

The line indicates linear regression line of best fit (dotted lines indicate $95 \%$ confidence intervals) implying that these variables are not significantly associated (for glyburide concentrations in this range); $R^{2}=0.008 ; P=0.464$. 


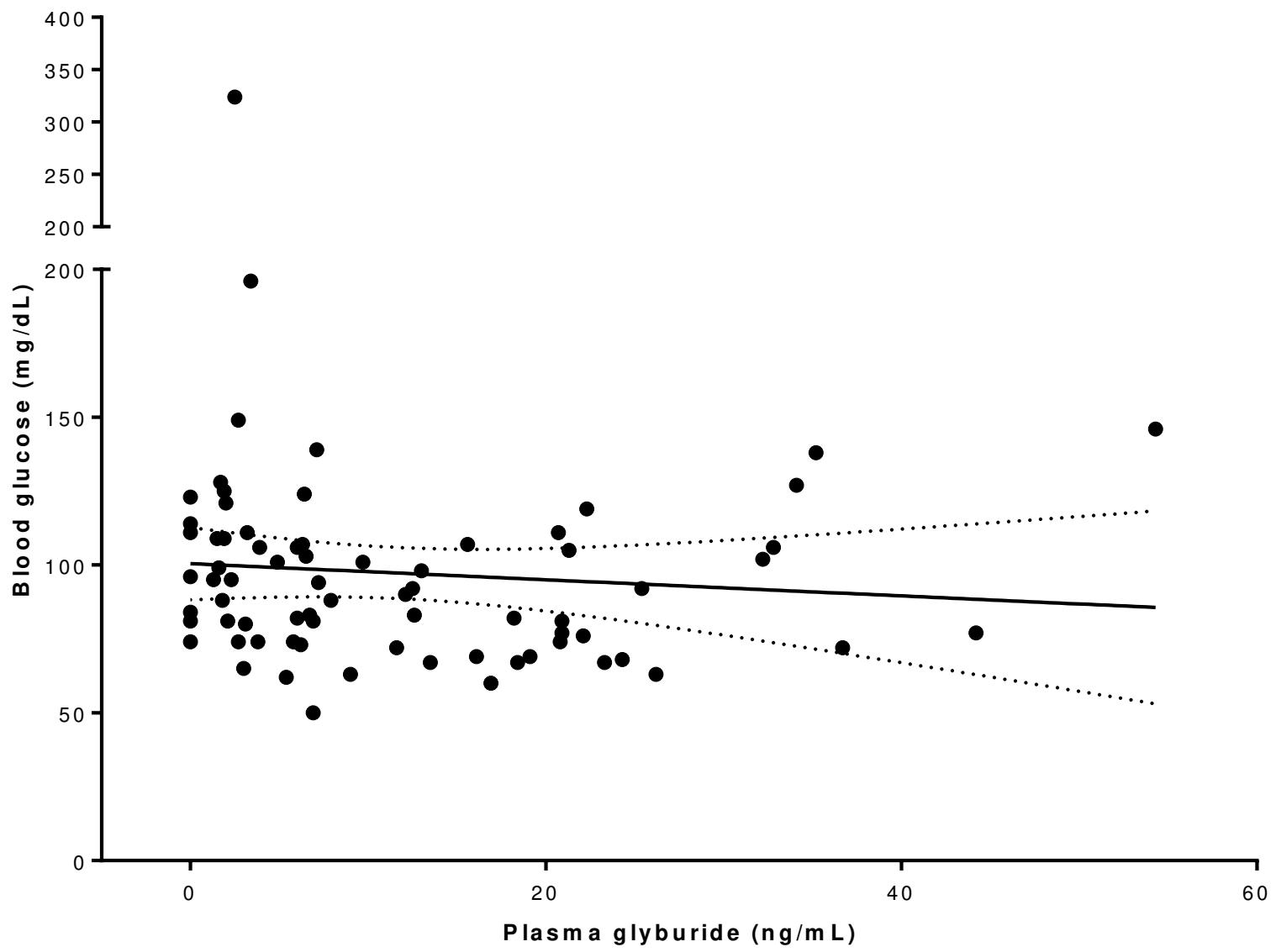


Figure 4 (on next page)

Predicted glyburide concentrations based on superpositioning of observed concentrations in individual dogs. Shaded area represents one standard deviation from mean. 


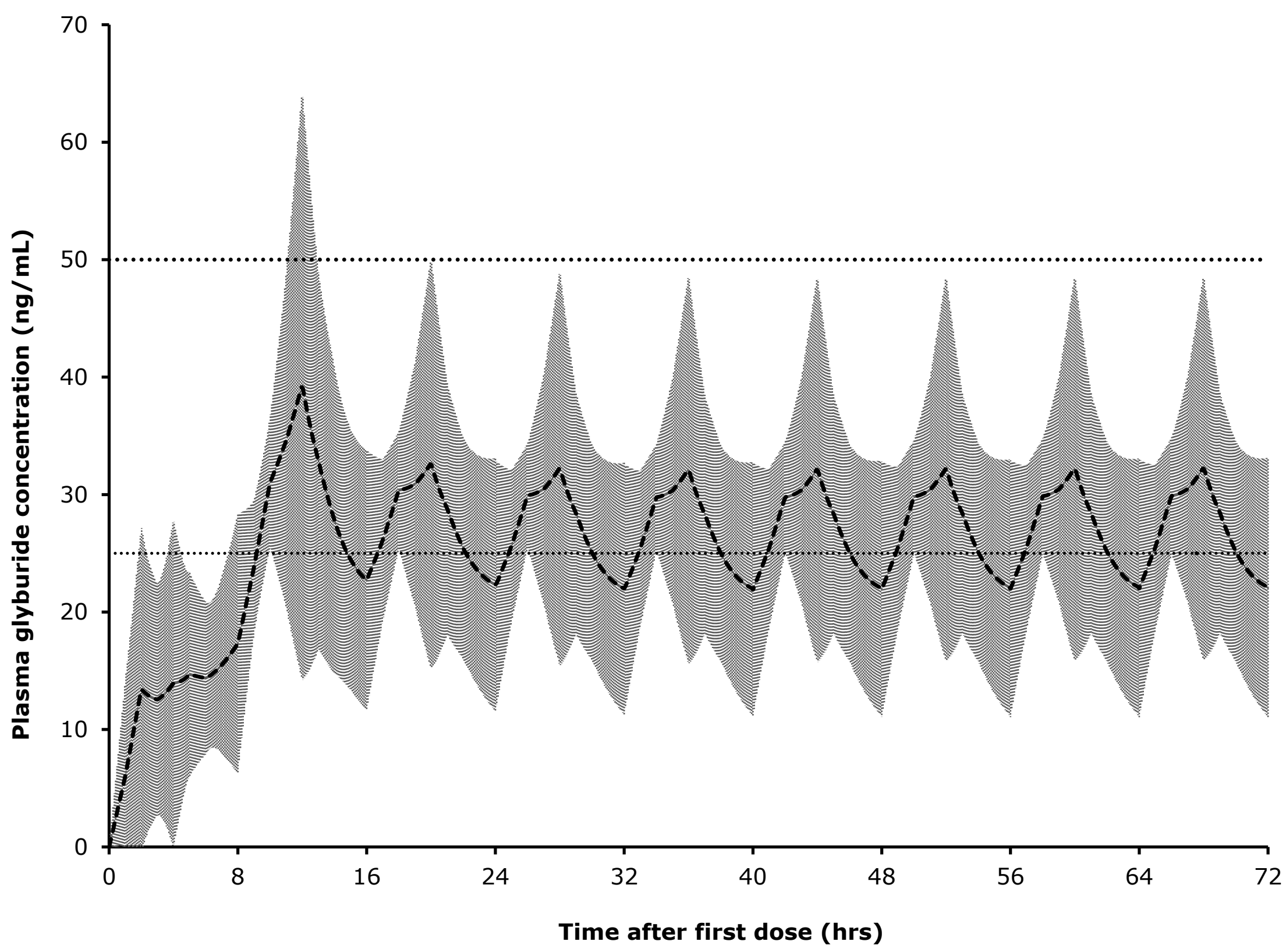

PeerJ reviewing PDF | (2017:11:21738:1:1:NEW 23 Jan 2018) 


\section{Table $\mathbf{1}$ (on next page)}

Estimates of pharmacokinetic parameters of glyburide in dogs $(n=6)$ based on noncompartmental and compartmental analysis of serum concentration after one oral dose of glyburide (75 mcg/kg) (see text for abbreviations; SD = standard deviation).

Notes: *See text for explanation about incomplete pharmacokinetic analysis of these dogs. †Onecompartment models with observed values weighted by $1 / \mathrm{y}^{2}$ and first order input and elimination fit best for Dogs 1-5; the model selected for Dogs 1, 2, and 5 included no lag time and assumption of K10=K01; the model selected for Dog 3 was similar with the exception of a lag time; the model selected for Dog 4 included no assumptions about K10 and no lag time. 
Table 1: Estimates of pharmacokinetic parameters of glyburide in dogs $(n=6)$ based on non-compartmental and compartmental

\begin{tabular}{|c|c|c|c|c|c|c|c|c|c|}
\hline Parameter & Units & Dog 1 & Dog 2 & Dog 3 & $\operatorname{Dog} 4$ & Dog 5 & $\operatorname{Dog} 6$ & Mean & SD \\
\hline \multicolumn{10}{|c|}{ Non-compartmental Analysis } \\
\hline $\mathbf{T}_{\max }$ & hr & 10 & 10 & 12 & 2 & 12 & 24 & 12 & 7 \\
\hline $\mathbf{C}_{\max }$ & $\mathbf{n g} / \mathbf{m L}$ & 20.9 & 26.2 & 54.3 & 32.2 & 44.2 & 9.7 & 31.3 & 14.7 \\
\hline$\lambda_{\mathrm{z}}$ & $/ \mathbf{h r}$ & 0.075 & 0.063 & 0.142 & 0.110 & 0.127 & $*$ & 0.103 & 0.030 \\
\hline $\mathbf{t}_{1 / 2 \lambda z}$ & hr & 10.9 & 10.9 & 4.9 & 6.3 & 5.4 & $*$ & 7.7 & 2.7 \\
\hline $\mathbf{A U} \mathbf{C}_{0 \text {-last }}$ & hr*ng/mL & 272.5 & 343.9 & 526.4 & 212.5 & 390.7 & 115.05 & 310.1 & 131.3 \\
\hline $\mathbf{A U C} \mathbf{C}_{0 \text {-inf }}$ & hr*ng/mL & 352.2 & 526.9 & 576.5 & 229.8 & 437.8 & $*$ & 424.6 & 124.0 \\
\hline AUC \% Extrap & $\%$ & 22.6 & 34.7 & 8.7 & 7.5 & 10.8 & $*$ & 16.9 & 10.4 \\
\hline $\mathrm{AUMC}_{0 \text {-last }}$ & hr*ng*ng/mL & 3611 & 4622 & 5750 & 1499 & 4968 & 1887 & 3723 & 1570 \\
\hline$A U M C_{0-\text { inf }}$ & hr*ng*ng/mL & 6583 & 11905 & 7308 & 2071 & 6469 & $*$ & 6867 & 3124 \\
\hline MRT $_{\text {last }}$ & hr & 13.3 & 13.4 & 10.9 & 7.1 & 12.7 & 16.4 & 12.3 & 2.8 \\
\hline MRT $_{\text {0-inf }}$ & hr & 18.7 & 22.6 & 12.7 & 9.0 & 14.8 & $*$ & 15.6 & 4.7 \\
\hline \multicolumn{10}{|c|}{ One-compartment Model $\uparrow$} \\
\hline $\mathbf{T}_{\max }$ & $\mathbf{h r}$ & 12.1 & 20.7 & 7.1 & 1.7 & 17.5 & $*$ & 12 & 7 \\
\hline $\mathbf{T}_{\text {lag }}$ & hr & - & - & 0.8 & - & - & $*$ & & \\
\hline $\mathrm{C}_{\max }$ & $\mathbf{n g} / \mathbf{m L}$ & 9.7 & 14.9 & 28.8 & 19.7 & 8.6 & $*$ & 16.4 & 7.4 \\
\hline $\mathbf{K}$ & $1 / \mathrm{hr}$ & 0.0829 & 0.0484 & 0.1566 & - & 0.0571 & $*$ & 0.0862 & 0.0426 \\
\hline K01 & 1/hr & - & - & - & 1.6933 & - & $*$ & & \\
\hline K10 & $1 / \mathrm{hr}$ & - & - & - & 0.1203 & - & $*$ & & \\
\hline $\mathbf{t}_{1 / 2} \mathrm{~K} 10$ & hr & - & - & - & 0.4 & - & $*$ & & \\
\hline $\mathbf{t}_{1 / 2} \mathrm{K01}$ & $\mathbf{h r}$ & - & - & - & 5.8 & - & $*$ & & \\
\hline $\mathbf{t}_{1 / 2}$ & hr & 8.4 & 14.3 & 4.4 & - & 12.1 & $*$ & 9.8 & 3.8 \\
\hline
\end{tabular}




\begin{tabular}{|c|c|c|c|c|c|c|c|r|r|}
\hline $\mathbf{A U C}$ & $\mathbf{h r} \mathbf{n g} / \mathbf{m L}$ & 317.7 & 837.7 & 500.6 & 200.5 & 411.0 & $*$ & 453.5 & 216.4 \\
\hline $\mathbf{V} / \mathbf{F}$ & $\mathbf{m L} / \mathbf{k g}$ & 2848.9 & 1850.7 & 956.4 & - & 3198.0 & $*$ & 2213.5 & 878.2 \\
\hline $\mathbf{C L} / \mathbf{F}$ & $\mathbf{m L} / \mathbf{h r} / \mathbf{k g}$ & 236.1 & 89.5 & 149.8 & 374.0 & 182.5 & $*$ & 206.4 & 96.3 \\
\hline
\end{tabular}

6 Notes: *See text for explanation about incomplete pharmacokinetic analysis of these dogs.

7 †One-compartment models with observed values weighted by $1 / \mathrm{y}^{2}$ and first order input and elimination fit best for Dogs 1-5; the

8 model selected for Dogs 1, 2, and 5 included no lag time and assumption of K10=K01; the model selected for Dog 3 was similar with

9 the exception of a lag time; the model selected for Dog 4 included no assumptions about K10 and no lag time. 